Cahiers de recherches médiévales

Journal of medieval studies

5 | 1998

Le choix de la prose (XIII ${ }^{\mathrm{e}} \mathrm{XV}$ e siècles)

\title{
Le clerc et le ménestrel
}

Prose historique et discours versifié dans le Perceforest

Michelle Szkilnik

\section{CpenEdition}

Journals

Édition électronique

URL : https://journals.openedition.org/crm/1412

DOI : $10.4000 / \mathrm{crm} .1412$

ISSN : 1955-2424

Éditeur

Honoré Champion

\section{Édition imprimée}

Date de publication : 30 octobre 1998

Pagination : 87-105

ISSN : 1272-9752

Référence électronique

Michelle Szkilnik, "Le clerc et le ménestrel », Cahiers de recherches médiévales [En ligne], 5 | 1998, mis en ligne le 01 octobre 2007, consulté le 15 décembre 2022. URL : http://journals.openedition.org/crm/ 1412 ; DOI : https://doi.org/10.4000/crm.1412

Ce document a été généré automatiquement le 15 décembre 2022.

Tous droits réservés 


\title{
Le clerc et le ménestrel
}

\author{
Prose historique et discours versifié dans le Perceforest ${ }^{1}$
}

\author{
Michelle Szkilnik
}

Quand au début du XIV e siècle, un auteur inconnu imagine de raconter l'histoire d'un roi d'Angleterre tout aussi inconnu, Perceforest, il choisit «naturellement » la prose. «Naturellement» parce que le Perceforest se veut la pré-histoire du monde arthurien, qu'il prétend donc se rattacher, comme l'avait fait L'Estoire del Saint Graal, à l'avant des grands cycles en prose du XIII ${ }^{\mathrm{e}}$ siècle, le Lancelot-Graal en particulier. Il opte donc pour le même mode d'écriture que les œuvres qu'il vient en quelque sorte préfacer. D'autre part, l'auteur veut donner à son roman le statut d'œuvre historique. Tout au long de son énorme somme, il déploie une extraordinaire habileté pour convaincre ses lecteurs qu'ils sont en train de se passionner pour de véritables chroniques du royaume d'Angleterre. Or depuis le début du XIII ${ }^{\mathrm{e}}$ siècle, en France, l'histoire s'écrit en prose ${ }^{2}$.

Pourquoi alors cette prose pseudo-historique est-elle brisée par l'intrusion du vers? Pourquoi toutes ces inscriptions rimées, ces pièces lyriques dont certaines peuvent être d'une longueur considérable? Que vient faire le vers dans une œuvre historique ? Sans doute les insertions lyriques ${ }^{3} \mathrm{du}$ Perceforest le rangent-elles dans une tradition romanesque bien illustrée au XIII ${ }^{\mathrm{e}}$ siècle. Est-ce à dire que le recours au vers contredit les prétentions historiques du Perceforest, dénoncent le caractère romanesque de l'œuvre? Il me semble que non. Le vers, réputé a-historique, mensonger, me paraît au contraire soutenir le projet du pseudo-historien, auteur du Perceforest. Ce n'est pas que le vers, corrigé de sa propension à mentir, circonscrit par la prose, soit mis vaille que vaille au service de l'histoire. Bien plutôt, il s'avère un mode de discours indispensable : il garantit la validité de l'histoire en lui donnant un sens, sens que la prose ne peut saisir ${ }^{4}$.

3 Le très long prologue au Perceforest mérite d'être examiné en détail parce qu'il est le lieu où l'auteur met en place la stratégie compliquée grâce à laquelle il espère établir le caractère historique de son œuvre ${ }^{5}$. Le sujet du roman est défini dans les premières lignes du texte: «mettre en escript ou langaige de France une ystoire d'un gentil roy qui jadiz regna en la Grant Bretaigne» (I, 61). Aussi simple que paraisse cette déclaration, elle pose déjà quelques questions : s'agit-il de mettre par écrit une version 
orale de l'histoire? S'agit-il de traduire « ou langaige de France » un ouvrage antérieur ? Si oui, un ouvrage en quelle langue? De quel roi est-il question? À quelle époque renvoie donc le «jadis»? Loin de nous éclairer immédiatement en indiquant ses sources, réelles ou fictives, et en racontant comment elles sont tombées entre ses mains, le narrateur choisit de différer toute explication, non sans promettre toutefois de revenir sur le sujet.

Si le narrateur du Perceforest décide de ne donner toutes ces informations que plus tard, ce n'est pas, comme on pourrait le craindre, pour éluder les questions, mais pour proposer d'abord la traduction de plusieurs œuvres antérieures : le début du Perceforest consiste en effet en un savant collage par lequel nous glissons subrepticement de textes réputés historiques à la fiction du roman. Le narrateur se lance pour commencer dans une longue description géographique de la Grande-Bretagne empruntée à deux historiens médiévaux: Orose et Geoffroy de Monmouth. Le plagiat est double, car non seulement la description est littéralement copiée des Historiae adversum Paganos d'Orose et de l'Historia Regum Britanniae de Geoffroy ${ }^{6}$; mais encore le narrateur du Perceforest s'inspire du plan de ses sources : il place sa présentation géographique au tout début de son ouvrage. En imitant la dispositio adoptée par tout bon historien médiéval ${ }^{7}$, il confère à son œuvre un caractère sérieux et savant.

5 La manière dont il utilise les descriptions de ses prédécesseurs vaut qu'on s'y arrête pour une autre raison. Au lieu en effet de traduire d'abord Orose puis de le compléter avec Geoffroy, le narrateur mélange les deux textes. La première phrase «Bretaigne la meilleur de toutes ysles est assise en la mer de Occean entre Gaule et Yrlande » vient de Geoffroy. La seconde, comme l'indique le narrateur lui-même, vient d'Orose: «Si comme dist Oroses, elle s'estend du long vers bise... » La troisième pourrait venir de l'un ou l'autre auteur puisque tous deux indiquent la dimension de l'île : huit cent mille pas de long sur deux cent mille de large ${ }^{8}$. La description des Orcades et de Thulé est traduite d'Orose mais la parenthèse sur le vent Chirrius semble un ajout du narrateur. Le long passage sur la richesse de la terre et les peuples qu'elle héberge vient de Geoffroy. Enfin la description de l'Irlande est tirée d'Orose et dans Historiae adversum Paganos, figure juste après celle de Thulé. En d'autres termes, le narrateur du Perceforest a farci le texte d'Orose d'un long passage de Geoffroy. Curieusement, il reconnaît à deux reprises sa dette, pourtant relativement modeste, envers Orose alors qu'il ne signale pas qu'il traduit Geoffroy de Monmouth.

6 Après la présentation géographique le récit historique s'engage sur un double repère temporel: la date de la fondation de Rome («sept cens et cinquante deux ans devant l'incarnation de Jhesuchrist») et celle du rapt d'Hélène, qui marque le début de la guerre de Troie ( quatre cens ans et trente devant la fondation de la tresnoble cité de Romme»). L'auteur indique cette fois qu'il traduit Darès le Phrygien'. Puis il retrouve Geoffroy pour relater la victoire d'Énée sur Turnus. A partir de là, il le suit à peu près fidèlement. Pourquoi l'auteur confesse-t-il ses emprunts à Orose et Darès mais n'avoue$\mathrm{t}$-il pas traduire Geoffroy? Serait-ce parce qu'Orose et Darès font figure de "vrais » historiens alors que l'œuvre de Geoffroy paraît plus romanesque, moins sérieuse? Vulgarisée par le Brut de Wace, l'Historia Regum Britanniae jouit d'un grand prestige pour avoir promu le roi Arthur au rang de héros mythique. Or ce n'est pas la partie arthurienne que Perceforest emprunte, mais le début de l'histoire des rois d'Angleterre, de Brutus au roi Pir. L'auteur du Perceforest craint-il que le nom de Geoffroy soit si 
fortement associé aux affabulations dénoncées par Wace ${ }^{10}$ que le sérieux de son entreprise en pâtisse? Redoute-t-il que l'ombre d'Arthur ne ternisse l'éclat de son propre héros, Perceforest, dont Arthur sera un lointain descendant ? La manière dont il traite une autre de ses sources laisse penser qu'il hésite à reconnaître ses dettes envers des œuvres romanesques : lorsque, pour introduire enfin ses personnages, il utilise, en les résumant très sommairement, les Vœux du Paon de Jacques de Longuyon, roman du début du XIV ${ }^{\mathrm{e}}$ siècle, bien connu sans doute quand est rédigé le Perceforest, il se garde bien là encore de confesser son emprunt. Orose et Darès sont d'anciennes et prestigieuses autorités latines. Mais ni Jacques de Longuyon ni Geoffroy de Monmouth ne semblent en mesure d'apporter le sérieux historique que réclame l'entreprise. Comment du reste faire confiance à un Geoffroy de Monmouth qui ignore, selon Perceforest, près de cent cinquante années de l'histoire de l'Angleterre? Cette lacune malheureuse, même si elle était inévitable comme va le montrer le narrateur plus loin, discrédite l'œuvre de ce prédécesseur sur qui il faut pourtant bien s'appuyer.

Dans son édition du premier livre de Perceforest, J.H.M. Taylor a montré combien toute la partie traduite de Geoffroy et d'Orose calque de si près le latin que le texte français en est parfois obscur et le plus souvent lourd et maladroit. La prose du prologue contraste de manière frappante avec la prose du roman proprement dit. Au début du $\mathrm{XIV}^{\mathrm{e}}$ siècle, la prose française a acquis une souplesse, une richesse qui la rendent apte à traduire le latin avec aisance. Le reste du Perceforest prouve que le prosateur sait manier avec art son outil. Pourquoi alors choisit-il de coller au latin dans son prologue? D'une part, on peut alléguer le souci de fidélité au texte originel qui se fait jour vers la fin du XIII siècle et s'amplifie au XIV ${ }^{\text {e11. }}$. D'autre part, ce décalque du latin qui rend le texte hermétique lui donne aussi un aspect savant. Perceforest ne se présente pas d'emblée comme un roman facile et plaisant mais comme une œuvre historique sérieuse et austère ${ }^{12}$. Le narrateur rappelle dès le début du prologue qu'un texte latin est à l'horizon de son texte français : «je commenceray ainsi que je le treuve en escript d'un preudomme qui du livre de latin le translata en nostre langaige» (PF I, p. 61). Le narrateur prétend ne pas être l'auteur de la traduction mais utiliser celle d'un "preudomme ", c'est-à-dire un sage, un érudit, un spécialiste qui s'exprime dans une langue savante et partant obscure. Le sérieux de la source, attesté par son jargon, rejaillit sur l'ensemble.

8 L'auteur du Perceforest entretient une sorte de flou autour de son travail dans le prologue : traducteur de Geoffroy de Monmouth, il refuse de nommer l'original latin, qu'il suit pourtant le plus souvent avec une extrême fidélité, fidélité qui garantit l'historicité du roman.

Ce flou qui témoigne sans doute de son embarras face à l'œuvre de Geoffroy, lui offre cependant quelques avantages : à la faveur de l'imprécision qui règne dans le prologue, l'auteur du Perceforest s'approprie des sources et des techniques qui appartiennent à l'original latin. Faisant allusion, par exemple, à la discorde qui éclata entre Lud et son frère Nennius, le texte français s'excuse de ne pas entrer dans le détail : «lequel debat pour ce que Gildas le hystoriographe le traitta assez prolixement sy esleuz je a trespasser, par quoy ce que si grant escripvain de si noble stile a traittié je ne soie veu honnir par dittié plus vil»(PF I, p. 86). Qui émet cette réserve? Qui refuse de rivaliser avec Gildas? Une rapide vérification dans l'Historia Regum Britanniae nous apprend que c'est Geoffroy lui-même ${ }^{13}$. L'hommage rendu à Gildas nous le suggérait déjà car nous savons que Gildas est l'une des sources de l'Historia. Mais un lecteur qui ne connaît pas 
Geoffroy et qui a oublié le «preudhomme »-traducteur dont il était question vingt pages plus tôt, attribuera la phrase au narrateur du Perceforest.

Un autre exemple de ce détournement est le recours aux incidences. Le récit chez Geoffroy tire son authenticité des rapprochements que l'écrivain britannique dessine habilement entre son histoire et l'histoire universelle, rappelant par exemple ce qui se passe en Judée ou en Grèce quand tel événement se produit en Grande-Bretagne. Geoffroy n'a pas inventé cette technique mais il l'utilise systématiquement. Perceforest conserve avec soin les rapprochements établis par son modèle : «En celui temps regnoit en Judee Hely le p[r]uvoire et l'Arche du Testament estoit prinse de Philistiens. Et aussi regnoient en Troies les filz de Hectoris, enchassez les successeurs Anthenoris. Et si regnoit en Ytalie Silvius Eneas filz de Enee et l'oncle Bruti, le tiers des Latins. » (PF I, p. 87). Il les souligne parfois plus nettement que l'écrivain anglais en les introduisant par le terme : «incidences $»^{14}$. Et surtout, il en insère d'autres. En une occasion, en effet, l'auteur du Perceforest abandonne Geoffroy pour emprunter de nouveau à Orose. Geoffroy ne s'étend guère sur le sac de Rome auquel se livrent les frères Brennius et Belinus. Jugeant sans doute que l'événement méritait plus qu'une mention rapide, Perceforest traduit la longue et détaillée description d'Orose, en indiquant sa source : «Oroses ou second livre en la fin » (PF I, p. 109). Il enchaîne sur un court emprunt au tiers livre d'Orose qui, dans le texte latin, ne suit pas la description du sac de Rome, et constitue une incidence au début du livre suivant: «En ce mesme temps que ceulx de Galles tindrent Romme, prindrent, embraserent et vendirent, Archaxerxes le roy des Prilans commanda par ses messaiges trestoute Grece a departir des armes et reposer en paix, denonçans celuy qui seroit au contraire de la paix estre requis par bataille » (PF I, p. 110). L'auteur du Perceforest multiplie ainsi les incidences, allant les chercher chez Orose quand il ne les trouve pas chez Geoffroy.

Quel est le but de cette stratégie compliquée qui à la fois cache et révèle ses sources ? Il s'agit de construire un cadre historique solide dans lequel va pouvoir s'inscrire la fiction du Perceforest. Mais le problème principal, exposé au début du prologue, n'est pas résolu: comment se fait-il qu'aucun historien avant l'auteur du Perceforest n'ait parlé de la brillante civilisation qui s'est épanoui pendant plusieurs générations en Grande-Bretagne? Le paradoxe est d'autant plus grand que, comme le rappelle l'écrivain, les chroniques d'Angleterre ont préservé le nom d'un grand nombre de rois sans importance sur qui il n'y a quasiment rien à dire sinon qu'ils ont régné à tel moment. Le narrateur se moque même du zèle excessif du chroniqueur qu'il suit (Geoffroy !) : « en leur temps firent (ces rois) pou de choses qui facent a recommander, ainçois fut cellui qui ce mist en escript tout ensonnié d'avoir leurs noms pour continuer sa matiere » (PF I, 120). Si donc un chroniqueur scrupuleux a pris soin de noter les noms de personnages sans importance, seul un véritable complot peut expliquer l'étonnant silence concernant Perceforest. Au livre VI, le narrateur explique en effet que le roi Scalpiol a systématiquement effacé tout souvenir de Perceforest, défendant qu'on en parle, que l'on écrive sur lui, édifiant même un château nommé Scalpiol sur les ruines du Franc-Palais ${ }^{15}$. L'histoire d'Angleterre a été amputée, falsifiée et c'est à l'auteur du Perceforest qu'il revient de rétablir une vérité occultée depuis des siècles. Et ne vient-il pas de donner dans son savant prologue les preuves incontestables de ses aptitudes d'historien? S'étant présenté comme un spécialiste de la carrure d'Orose, il peut prétendre corriger l'histoire, à l'aide des nouvelles sources que le hasard a fait réapparaître. 
Ce n'est donc qu'après une soixantaine de pages d'exposé historique que le narrateur met enfin en place la fiction du livre originel trouvé dans une providentielle armoire ${ }^{16}$. Mais là encore il prend soin d'enrober son invention dans une série d'événements historiques vérifiables, et d'autant plus aisément vérifiables qu'il s'agit d'événements contemporains de la rédaction du Perceforest. Les sources sensationnelles que l'auteur du Perceforest donne à lire au public viennent juste d'être exhumées. Ce second prologue $^{17}$ (qui est le véritable dans la mesure où c'est à cet endroit que l'écrivain expose les conditions de production de son ouvrage), évoque d'abord le mariage d'Édouard d'Angleterre et d'Isabelle de France, dont il donne la date précise : «l'an de l'Incarnation de Nostre Seigneur mil trois cens et sept, le jour de la Purification de la benoite Vierge Marie» (PF I, 120) ${ }^{18}$. Cette date chrétienne fait pendant à la date païenne qui ouvrait l'exposé historique: «Quatre cens ans et trente devant la fondation de la tresnoble cité de Rome» (PF I, 63). Le nouveau repère sur l'axe du temps est la naissance du Christ et non la fondation de Rome. En passant aisément de l'un à l'autre, le narrateur prouve sa maitrise des deux calendriers et de nouveau ses qualités d'historien. Toutefois si la date païenne est importante puisqu'elle marque le début de la guerre de Troie, la date chrétienne n'a pour fonction que de situer dans un passé très récent la découverte du fameux manuscrit. Le mariage n'intéresse le lecteur que dans la mesure où il fournit la raison du voyage que le comte de Hainaut entreprend en Angleterre. Ce détail inutile et secondaire fait pourtant l'objet d'un développement d'une vingtaine de lignes, alors qu'il n'était pas même besoin de justifier la présence du comte en Angleterre. La date du mariage, officielle, connue de tous, sert à garantir tout le développement qui suit et qui n'a pourtant quasiment rien à voir avec elle. ${ }^{19}$

Le narrateur nous offre une deuxième garantie: le comte de Hainaut dont il est question est un personnage historique: Guillaume 1er, mort en $1337^{20}$. Or ce serait Guillaume qui aurait rapporté d'Angleterre le manuscrit du Perceforest et aurait ordonné à un moine de l'abbaye de Saint Landelin à Crespin de le traduire du latin en français. La réalité historique de Guillaume et de l'abbaye de Crespin engage le lecteur à croire que l'abbaye de Wortimer, où aurait été découvert le manuscrit, et son accueillant abbé ont réellement existé aussi, ce qui n'est pas le cas.

Bien d'autres détails concourent au même effet : l'abbaye de Wortimer porte le nom de son fondateur Wortimercius, le Vortimer de l'Historia Regum Britanniae, qui réapparaît dans le livre VI, lorsque le narrateur du Perceforest renoue en partie avec la chronique de Geoffroy. Par ailleurs, l'abbé prétend avoir découvert à côté du manuscrit une couronne d'or ${ }^{21}$ qu'il aurait envoyée au roi Édouard : que le lecteur sceptique aille vérifier auprès d'Édouard! Quant au clerc qui aurait traduit la chronique de grec en latin, il venait de Paris où il étudiait la philosophie mais n'avait pu y rester parce qu'il était accusé d'homicide. Ce détail rapproche le clerc de Brutus : les deux hommes sont exilés pour avoir commis un meurtre et échouent en Angleterre. ${ }^{22}$ D'autre part, puisqu'il s'agit d'un épisode supposé récent, il joue le même rôle que l'évocation du mariage d'Édouard: créer des effets de réel pour rendre plausible la séquence d'événements qui font éclater la vérité cachée pendant si longtemps.

La précision des détails secondaires égare le lecteur et le détourne d'examiner plus à fond la transmission du texte. Or cette transmission est loin d'être claire : l'abbé de Wortimer a trouvé un manuscrit dans une armoire dissimulée dans le mur d'une tour elle-même à moitié enfouie dans le sol ${ }^{23}$. Il a conservé pendant une dizaine d'années le manuscrit qu'il ne pouvait déchiffrer car il n'en connaissait pas la langue. Le clerc grec 
homicide accepte de le lui traduire de grec en latin mais apparemment n'a pas complété son entreprise : «le Gregoiz ay eu (c'est l'abbé qui parle) ceans plus de demy an si ne m'en a que cecy translaté » (PF I, p. 123). L'abbé accepte cependant de confier le livre au comte qui le fait copier en moins d'un mois. Mais qu'est-ce qui est copié : la traduction latine ou l'original grec? Le texte emploie à deux reprises le pronom «cestuy » mais sans que l'on sache à quoi il renvoie exactement. On est tenté de croire que c'est la traduction latine qu'a fait copier le comte puisque de retour en Hainaut il demande à un moine de l'abbaye de Saint Landelin de «translater de latin en françois» l'ouvrage. Mais cela signifie-t-il alors que nous n'avons pas l'intégralité de l'œuvre? Guillaume s'étant intéressé à d'autres entreprises, le moine de Crespin chargé de la traduction a laissé traîner l'ouvrage, ce qui a retardé encore la parution du livre.

Ainsi se clôt le prologue sans nous révéler l'identité du narrateur qui est apparu au tout début du Perceforest. La seule certitude, c'est qu'il n'est pas le moine de Crespin. Il ne serait donc ni le traducteur du prologue historique ni celui du reste du texte. Pourtant on a vu que la formule qui ouvrait le roman "mettre en escript ou langaige de France " suggérait une activité de traducteur. Le narrateur reste ainsi très vague sur son rôle, il s'abrite derrière une série d'intermédiaires mal connus : un clerc grec, un moine ami de Guillaume, un "preudome» dont l'anonymat suspect est oblitéré par l'abondance de noms propres secondaires: sources de moindre importance que le narrateur met pourtant en avant, Orose, Darès le Phrygien, Gildas; ou personnages qui n'interviennent pas dans la production du texte: Édouard, Guillaume. Or, loin de s'arrêter là dans son entreprise de mystification, l'auteur de Perceforest complique encore sa stratégie dans le cours du roman.

Perceforest est divisé en six livres. Nous venons de voir que le premier est précédé d'un important prologue qui vise à ancrer le roman à la fois dans l'histoire d'Angleterre et dans l'histoire universelle et à convaincre le lecteur de l'authenticité de ce qu'il va lire. Chacune des cinq autres parties possède aussi une courte préface qui paraît n'avoir pour fonction que de justifier la division en volumes ${ }^{24}$. Le narrateur qui y prend la parole semble être le même que celui qui s'adresse au lecteur au tout début du roman. Il explique que «il est de necessité de faire aucunes fois plus d'un ou de deux volumes parlans d'une mesme matiere» (PF, III-1, p. 1). La matière est longue, nous dit-il. Tout ne peut tenir en un seul livre. Aussi prend-il sur lui de découper l'histoire et de donner des titres aux différents volumes, titres du reste plutôt évidents: "Pour ce ay je encommencé le tiers livre [...] lequel nous nommerons le tiers livre des Anciennes Chroniques d'Angleterre ». Le narrateur serait donc tout au plus une sorte d'éditeur ${ }^{25}$. C'est à lui que nous devrions la division en chapitres et les titres de ces derniers. Ce rôle serait conforme à celui qu'il s'assigne dans le premier livre où il prétendait ne faire que recopier différentes traductions dans un ordre chronologique.

Les préfaces aux livres II à VI nous apportent toutefois une information nouvelle. Elles introduisent un nom propre: les «Anciennes Chroniques d'Angleterre » ont été «jadis compillees par ung bon clerc nommé Cressus qui estoit de l'ostel du tresexcellent prince Alexandre le Grant, lequel par bon advis lui bailla avecques plusieurs autres roys charge de ce faire. ${ }^{26}$ Ce Crésus apparaît en réalité dès la fin du premier livre. Les dames de la forêt Darnant souhaitent que tous les hauts faits accomplis par Perceforest et ses compagnons contre l'infâme lignage de l'enchanteur Darnant soient gardés en mémoire. Alexandre renchérit et s'engage à confesser le premier tout ce qui lui est 
arrivé dans la forêt à la condition qu'en fassent autant les chevaliers qui avaient participé à la quête de Bétis-Perceforest. Le roi Perceforest fait alors appeler

«un sien clerc nommé Cresus, sage, discret et preudomme, si lui dist : «Cressus, il fault que vous aiés parchemin et encre et quant Alexandre vous huchera vous soiés prest pour mestre par escript ce qu'il vous devisera et pareillement ferez aux autres nœuf et en general de toutes les adventures qui sont advenues en cestuy paÿs depuis que le noble empereur y est venus et nous aussi, et non point seulement ce qui est advenu jusques a present mais ce qui advendra d'ores en avant en Angleterre de jor en jor.» En la maniere que le bon roy le commanda, Cressus le bon clerc s'i emploia et ce que nous en sçavons nous vient de lui. Car selon la relation des nobles barons, il mist par escript toutes leurs adventures, au mains celles qui estoient dignes de memoire.» (Ars. 3484, fol. 403r²).

19 De loin en loin, le roman réassigne à Crésus la fonction de consignateur des hauts faits $^{27}$. Le livre VI précise la manière dont Crésus a recueilli les événements à consigner : il a entendu les dépositions sous serment de tous les chevaliers : "Il avoit ung clerc nommé Cresus, auquel tous les chevaliers du roiaulme, en especial ceulx du Franc Palaiz, et le roy meismes qui tant fu preu de son corps, venoient dire sur leur serement par chascun an tout ce que advenu leur estoit en leurs adventures. (Ars. 3493 , fol. $\left.198 \mathrm{r}^{\mathrm{e}}\right) .{ }^{28}$

20 La mise en scène que l'auteur du Perceforest imagine n'est guère originale ; elle rappelle celle des grandes sommes en prose du XIIIe, où l'on voit les chevaliers raconter leurs aventures après avoir juré de dire toute la vérité, et les clercs coucher leurs récits par écrit $^{29}$. Le terme de chronique toutefois et l'insistance de Perceforest qui veut que Crésus y inscrive ce qui arrivera "de jor en jor» arrêtent l'attention. Malgré leurs prétentions historiques, la Queste del Saint Graal ou La Mort le roi Artu ne proposent pas une chronique au jour le jour du règne arthurien. Par ailleurs, l'idée de hiérarchiser les différents "contes", de diviser la matière en "branches" suggère un modèle d'organisation tout à fait différent de celui de chroniques, un modèle non seulement plus complexe mais encore investi d'un sens qui transcende les simples problèmes de présentation matérielle. Toutes les aventures sont subordonnées au haut conte du Graal qui les illumine d'un éclat surnaturel. ${ }^{30}$ Rien de tel semble-t-il dans Perceforest. Son modèle ne serait-il pas plutôt ici les Grandes Chroniques de France dont le Perceforest serait comme le pendant anglais, un pendant qui aurait l'avantage de l'antériorité ? Bien avant que le roi de France Louis IX charge les moines de Saint-Denis de rédiger en français les Grandes Chroniques de son royaume, le roi d'Angleterre Perceforest aurait enjoint à son clerc de compiler les "Anciennes Chroniques d'Angleterre", entreprise historique de la même envergure que celle lancée par saint Louis. Après la mort de Crésus, sa fonction passe à d'autres clercs. On peut tout de même s'étonner de la longévité du personnage qui apparemment survit à l'invasion romaine puisqu'il a également compilé le livre IV qui relate la défaite du Franc-Palais.

21 En confiant à un clerc imaginaire, supposé contemporain des aventures, la charge de les narrer, l'auteur du Perceforest n'invente rien. Toutefois en choisissant d'appeler son récit «Chroniques d'Angleterre », il confère clairement à son roman le statut d'œuvre historique. Alors pourquoi recourt-il si abondamment au vers? J. Lods a calculé que le roman contient plus de deux mille vers. La place réservée au discours poétique dans ce qui se veut une chronique peut paraître singulière, voire paradoxale. Car si le vers transmet bien une certaine vérité, on s'accorde à penser que c'est une vérité qui n'est justement pas d'ordre historique ${ }^{31}$. Or il me semble que dans le Perceforest, prose et vers 
ne s'opposent pas mais se renforcent: le vers sert le projet historique au lieu de lui nuire. À la chronique engluée dans le quotidien, dans une immédiateté sans horizon, le vers apporte une hauteur de vue, une perspective véritablement historique ${ }^{32}$. Le discours poétique oriente les événements du Perceforest, les inscrit sur un axe dont la direction ne peut apparaître clairement au brave chroniqueur Crésus notant au jour le jour les faits marquants des règnes successifs.

L'intrusion du vers dans Perceforest prend des formes différentes. On rencontre un grand nombre de pièces que le texte qualifie de lais et qui présentent en effet toutes les caractéristiques des lais lyriques du Tristan en prose. Compositions élaborées, en général assez longues, ces lais sont le plus souvent l'œuvre d'un personnage important du roman. S'y ajoutent des prières adressées aux différents dieux; elles n'ont pas la même fonction que les lais mais elles possèdent également une forte valeur affective. Enfin, le roman est semé d'inscriptions versifiées, un simple couplet parfois, parfois une dizaine de vers, dont l'auteur reste mystérieux. Ces trois types de discours poétique jouent chacun un rôle dans le savant système qui garantit l'historicité de la prose.

Ce sont les lais lyriques ou les chansons qui ont surtout retenu l'attention des critiques successifs. J. Lods les a édités et en a par ailleurs souligné l'importance dans sa longue étude du roman ${ }^{33}$. On pourrait alléguer que l'auteur du Perceforest ne fait que céder à une mode inaugurée par les romans du XIII ${ }^{\mathrm{e}}$ siècle, Guillaume de Dole d'abord, puis le Tristan en Prose. De fait, Perceforest cherche clairement à rivaliser avec les grandes sommes du siècle précédent. De même que Tristan rivalisait avec le cycle du LancelotGraal, l'imitant et le renouvelant, Perceforest emprunte les techniques de ses prestigieux prédécesseurs et les remodèle à sa façon, comme on l'a vu pour son prologue. Mais cette réécriture s'avère extrêmement complexe et ne peut se justifier par le seul désir de rivaliser avec les textes du XIII ${ }^{\text {e }}$ siècle. Il convient d'abord de prendre en compte la diversité des lais et des chansons: les circonstances dans lesquelles ces pièces sont composées, puis interprétées, varient grandement.

Le Lai piteux par exemple est l'œuvre de la belle Néronès-Cuer d'Acier qui y raconte sa tragique histoire et qui la chante elle-même devant son ami Nestor et la reine-fée, mère de ce dernier. Il semble y avoir simultanéité entre la composition et l'interprétation. D'autre part, le lai ayant à peu près rempli sa fonction, à savoir révéler, à la reine fée au moins, l'identité de la jeune interprète, l'exécution à laquelle s'est livrée Néronès a pu rester unique.

En revanche, le Lai de la Rose raconte une aventure arrivée à des inconnus; on apprend au livre $\mathrm{V}$ que l'auteur en est le père de Ponchonnet, le roi des ménestrels à la cour de Perceforest ${ }^{34}$; les dames l'interprètent à l'occasion de grandes fêtes pour mettre en garde les chevaliers trop hardis. Les interprètes «modernes» n'ont aucun lien avec les personnages évoqués dans le lai. Celui-ci se transmet de génération en génération, de ménestrel en ménestrel et donne lieu à des interprétations répétées. Si le Lai Piteux ressemble à ceux que l'on trouve dans le Tristan en prose, le Lai de la Rose évoque plutôt la technique du Guillaume de Dole. Sans doute ne s'agit-il pas d'un poème rapporté ; il est presque certain qu'il est bien l'œuvre de l'auteur du Perceforest. Il n'en demeure pas moins qu'il fonctionne comme les chansons que le trouvère Jouglet, ou d'autres ménestrels, exécutent pour l'empereur Conrad et sa cour.

D'autres pièces peuvent donner lieu à plusieurs interprétations, par le créateur luimême d'abord, puis par un «professionnel ». Lyonnel, amoureux de Blanchette, la fille 
de la Reine-fée, compose le Lai de Complainte dans lequel il expose son désespoir de ne pouvoir se présenter devant son amie et devant la reine parce qu'on lui a dérobé les preuves des prouesses qu'il avait accomplies pour mériter Blanchette. Or un ménestrel l'entend chanter son lai et l'exécute à son tour devant Blanchette. Celle-ci y répond par le Lai de Confort que le ménestrel ira chanter à Lyonnel. Il en va de même du Lai secret composé par Blanchette, Priande et Liriope pour engager leurs amis, Lyonnel, Estonné et Le Tor à venir leur rendre visite. Les trois jeunes filles chargent une demoiselle de transmettre leur message musical (PF III-1, 237). La demoiselle vient exécuter ce lai nouveau devant le roi Perceforest et sa cour lors de la fête du Chastel Desvoyé (PF III-1, pp. 274-78). Plus tard, le ménestrel Ponchonnet la rencontre et lui demande de lui enseigner ce lai qu'il pourra à son tour chanter pour Lyonnel (PF III-2, pp. 13-15). Le ménestrel joue le même rôle que le harpeur qui va et vient entre Iseut et Kahedin et recueille le lai mortel du jeune chevalier dans le Tristan en prose ${ }^{35}$. Quand la communication directe s'avère impossible, il maintient pourtant le lien entre les personnages. Bien que le père de Ponchonnet se voie attribuer la composition du Lai de la Rose, la principale fonction que Perceforest assigne au ménestrel est celle de greffier : il recueille les lais, il les mémorise pour pouvoir les transmettre et porter témoignage.

J'aimerais m'attarder sur une dernière pièce parce que l'histoire de sa création et de sa transmission met particulièrement bien en valeur le rôle de la poésie et la fonction du ménestrel dans le roman. Il s'agit du Lai de Pergamon. Ce lai a été composé par le vieil ermite Pergamon lui-même, comme l'indique la première strophe. Ou, plus exactement, il s'agit d'une composition collective puisque Pergamon, à l'article de la mort, n'a pu achever son œuvre et a prié ses douze petites-filles de la mener à bien. Chacune d'elles à son tour contribuera donc à l'ensemble. Le lai célèbre les exploits des Chevaliers aux douze vœux, tels qu'ils ont déjà été décrits, en prose, à la fin du livre I. Dans les dernières strophes, il est aussi rappelé que l'ermite, avant de mourir, a organisé un ensemble de douze tournois durant lesquels chacun des douze chevaliers pourra, s'il se montre preux, gagner la main de l'une des demoiselles. Intimement lié à l'histoire, ce lai est chanté à l'occasion de chacun des douze tournois. Chaque fois, le frère des jeunes filles, également nommé Pergamon, explique les circonstances de la création du lai et pourquoi il est nécessaire de le chanter :

"Seigneurs, dames et damoiselles, il me samble bon et convenable qu'on declaire pourquoy ceste feste et les autres qui sont passees, et aussi celles qui sont a venir, furent faittes et estorees, afin que tous hommes et femmes qui n'en sont point encores advertis le sachent. Sy vous plaise tous et toutes de faire silence et vous orrez ung lay que Pergamon le bon ancien preudhomme jadis fist, lequel Pergamon fut grand-pere de ces huit pucelles et des quatre roynes qui sont illec assises a celle seconde table que vous pouez veoir. [...] Or vous plaise doncques entendre le lay que le noble hermite compila a l'ayde des douses pucelles ses niepces. » (PF III-2, p. 6) ${ }^{36}$.

Pergamon fait alors s'avancer le roi des ménestrels qui chante le lai en s'accompagnant de la harpe.

Mais il ne s'agit pas simplement de justifier chaque tournoi en évoquant les dernières volontés de l'ermite. Le lai peut en effet être interprété dans d'autres occasions. La messagère chargée d'exécuter au Chastel Devoyé le Lai secret par exemple, joue d'abord «le Lay du Gentil Hermite» (PF III-1, p. 274). Les exploits des douze chevaliers continueront d'être célébrés longtemps après que chacun d'eux aura conquis par sa prouesse l'une des petites-filles de Pergamon, longtemps même après leur mort. Ainsi Ourseau, petit-fils de la Reine-fée, voit-il en rêve un ménestrel harper le Lai de 
Pergamon, avant de rencontrer en effet le harpeur et de l'entendre jouer le lai (PF, IV-2, p. 916). Ponchonnet, dont la longévité égale celle du clerc Crésus, chante encore ce lai au mariage de Gallafur et d'Alexandre-Fin-de-Liesse. ${ }^{37}$ Comme chez Marie de France, le lai a donc pour fonction de conserver la mémoire de hauts faits dignes d'être connus et glorifiés, génération après génération. Le Lai de la Rose préserve le souvenir d'une anecdote curieuse dont la singularité et l'exemplarité méritent d'être retenues. Le Lai de Pergamon sauve de l'oubli un passé national prestigieux, où les générations futures puiseront l'énergie de se battre pour leur indépendance. Il élève à la dignité de héros mythiques les douze chevaliers. C'est sur ces mythes fondateurs que les chevaliers adoubés après le massacre du Franc-Palais s'appuieront pour reconstruire une brillante civilisation.

Le rôle de la poésie est donc double : d'une part, elle assure la continuité de l'histoire en transformant celle-ci en mythes producteurs d'un nouveau départ, d'une nouvelle histoire ${ }^{38}$. D'autre part, son mode de transmission lui assure la survie et du même coup garantit la survie du passé. En effet, quand la totale destruction du Franc-Palais fait craindre la disparition des archives, les lais mémorisés par les ménestrels conservent intact le passé. Le ménestrel joue donc un rôle essentiel car autant que le clerc, il est la mémoire de son époque, et des époques antérieures. Crésus, le clerc chroniqueur, a scrupuleusement noté tous les événements du règne de Perceforest. Mais quand les sombres présages sur l'avenir du pays se multiplient, le roi juge bon de cacher ses trésors et ses archives. Dans une «charte» accompagnant sa couronne et le manuscrit des chroniques, le roi fait savoir à ses successeurs que pour préserver sa mémoire et celle de ses contemporains, il a volontairement dissimulé «le grant livre ou toutes les adventures qui sont a recorder qui advindrent a moy et a tous les gentilz hommes du roiaulme, sont mises, dés le commenchement de mon regne jusques a la destruction " (Ars. 3493, fol. $179 \mathrm{v}^{\mathrm{o}}$ ). ${ }^{39}$. Ces documents vont rester inaccessibles pendant une génération. Dans l'intervalle, ce sont les ménestrels qui racontent aux fils des héros de première génération les exploits de leurs pères. Galopin, fils de Ponchonnet, explique à Ourseau que lui aussi a été gratifié d'une vision:

«il me sambloit que mon pere me dist ainsy: «Filz Gallopin, cesse ton somme, sy t'en va atout ta harpe en la Forest Darnant et la recorde les lais du tamps passé que je t'aprins en ta jennesse, parquoy recorder tu puisses les prouesses des anciens preudhommes devant la destruction de Bretaigne : le temps en est venu. » Sy m'en levai et accorday ma harpe, puis m'en vins vers ceste fontaine ou j'encommençay a jouer le Lai de l'Ermite ainsi que mon pere me le commanda. Or vous estez embatu sur moy, dont moult suis joyeux, car depuis la destruction de Bretaigne je n'avoie veu chevalier.» (PF, IV-2, pp. 917-18).

31 L'histoire se transmet oralement et par le biais de la poésie car si Galopin, en sa qualité de témoin, peut raconter en prose les «merveilles qui avindrent au Francq Palais » et vanter les mérites «de la chevallerie qui y repairoit au tamps du roy Perceforest ", en revanche, il n'est pas contemporain des événements racontés dans le Lai de Pergamon. La connaissance qu'il en a lui vient de son père qui avait lui-même chanté le lai composé par l'ermite. Le roi des ménestrels que le petit-fils de l'ermite invite à harper lors des douze tournois est en effet Ponchonnet ${ }^{40}$.

Comme le clerc, le ménestrel a donc la responsabilité du passé. Il doit apprendre les lais et les interpréter de manière à transmettre la mémoire d'un peuple. Le narrateur s'intéresse moins à l'instant de la création, qu'aux différents et nombreux moments de la «performance » (au sens linguistique du terme), car ce sont eux qui consolident son 
entreprise historique. Ils rendent compte de la transmission du passé et participent de la complexe stratégie d'authentification mise en place dans le roman. ${ }^{41}$ Le rôle du clerc et celui $\mathrm{du}$ ménestrel finissent $\mathrm{du}$ reste par se confondre. ${ }^{42}$ Le roi Gallafur, plein d'admiration pour l'entreprise dont son aïeul Perceforest avait chargé le clerc Crésus, décide de faire également rédiger des chroniques de son règne. Or il confie la tâche au fils de Ponchonnet, qui s'appelle à présent Ponchon. ${ }^{43}$ Est-ce un autre fils ou Galopin qui a changé de nom? Aurait-il changé de nom en changeant de fonction? Ce fils du roi des ménestrels est en tout cas bien choisi pour mener à terme le travail de rédaction de chroniques ${ }^{44}$. De même quand, au livre VI, le roi Scapiol décide d'éradiquer le souvenir de Perceforest et de son lignage, il s'en prend en particulier aux ménestrels dans leur double activité de poètes et d'historiens : « Meismes aux menestrels fist il deffendre sur paine de mort que jamais histoire, contes, laiz ne fables ne fussent de lui (= Gallafur) recordez mais mis du tout en oubly et en neant, et deschiriez se trouvez fussent en escript.» (Ars. 3493, fol. 331rº).

Mais le clerc-ménestrel ne se contente pas d'enregistrer le présent et de rappeler le passé. Il joue un rôle actif dans l'avènement du nouveau monde. Il assiste le destin en veillant à ce que chacun accomplisse la mission qui lui est réservée. Ponchonnet par exemple prévient la fille de Remanant de Joie qu'elle doit se rendre au perron merveilleux où une aventure exceptionnelle va se produire, aventure dont elle sera l'héroïne (PF IV-2, p. 1016). Après que tous les nouveaux chevaliers, fils des héros de deuxième génération, ont assisté à l'aventure du perron, Ponchonnet intervient encore une fois pour guider les jeunes chevaliers et permettre la naissance d'une société digne de celle qui a été détruite à la bataille du Franc-Palais : il exhorte les chevaliers à se donner un roi et quand ceux-ci s'interrogent sur la descendance de Perceforest, il les engage à rechercher la fille de Remanant-de-Joie et de Béthoine, petite-fille de Perceforest et d'Alexandre. En attendant que le jeune Lyonnel la retrouve, il conseille de différer l'élection du roi et annonce une série de tournois qui doivent se tenir à la Fontaine aux Pastoureaux (PF IV-2, pp. 1132-38), introduisant ainsi ce qui sera en grande partie la matière du livre $\mathrm{V}$. Ansi assume-t-il une responsabilité à la fois dans la fiction, en donnant à la nouvelle société chevaleresque des règles héritées de la société précédente, et dans la production du texte en maintenant et soulignant la continuité et la cohérence du récit. La structure du livre $\mathrm{V}$ calque en effet celle du livre III.

Cette troisième fonction que le roman prête au ménestrel est aussi celle du discours poétique. La poésie n'ouvre pas seulement sur le passé. Elle autorise l'écriture de l'histoire parce qu'elle est également ouverture sur l'avenir.

Pour intéressants qu'ils soient, les lais et chansons ne constituent qu'un des types du discours poétique dans le roman. Un autre, très représenté, est l'ensemble des inscriptions qui apparaissent mystérieusement dans le royaume de Perceforest. On peut en rapprocher les énigmatiques paroles en vers proférées par une statue, ou quelque tête étrange, comme celle de Vermineux. Leur caractéristique est de n'avoir aucun auteur manifeste. Alors que l'on sait qui a composé tel lai, les inscriptions paraissent se graver d'elles-mêmes sur le support qu'elles ont choisi. Quand il s'agit d'une déclaration, elle a beau émaner d'un endroit précis, son origine reste mystérieuse. Qu'est-ce qui fait parler la tête de Vermineux : un esprit mauvais? l'âme de l'ancien chevalier? Et que dire de l'étrange statue du Chevalier au Dauphin? La voix entendue par les jeunes filles et par Ourseau vient-elle de «l'ymaige» ou d'ailleurs? Le jour qui tombe ne permet pas de se prononcer (PF, IV-2, p. 914). 

qui est la "pucelle» enlevée par le roi. Elle pose donc une énigme aux générations à venir qui doivent reconstituer l'histoire. Elle rappelle toutes les autres inscriptions que les dames de la forêt, premières historiennes du royaume de Grande-Bretagne, apposent sous les étranges statues de chair qu'elles ont érigées en souvenir des méfaits de Darnant et ses hommes ${ }^{46}$. Mais le fait qu'elle soit composée en vers par un "preudhomme", un ancêtre des ermites de la Queste del Saint Graal, ${ }^{47}$ la différencie pourtant des inscriptions en prose rédigées par des femmes magiciennes. Une autre épitaphe lui fait pendant dans ce livre III et atteste plus clairement la nouvelle nature de ce "genre littéraire»: l'épitaphe sur le tombeau de la reine Flora, épouse du magicien Aroés. Celle-ci en effet n'est attribuable qu'à Dieu lui-même, le «Souverain Plasmateur ", qui a frappé le faux dieu et honoré sa femme vertueuse. Parce que Dieu est l'auteur du texte, celui-ci est meilleur que celui du «preudhomme» ermite. Bien qu'elle résume elle aussi l'histoire, l'inscription reste en effet compréhensible pour la postérité qui sans doute ignorera tout de l'intervention de Gadiffer et Flamine mais apprendra l'essentiel, à savoir que c'est Dieu qui a provoqué la chute du magicien. Lorsqu'Ourseau, une génération plus tard, découvre le tombeau de la reine, il comprend immédiatement l'histoire et admire l'efficace brièveté du poème: «la gisoit grant histoire en pou de vers » (PF IV-2, p. 814). Par ailleurs, à la différence de l'épitaphe du roi Fergus, celle de la reine Flora ouvre aussi sur l'avenir. Elle nous renseigne sur le sort des personnages après leur mort: Aroés est aux enfers, "en la caverne obscure », Flora «es cieulx ». CEuvre de Dieu, l'épitaphe de la reine Flora place tout l'épisode de la Roide Montaigne dans une perspective historique, voire ici eschatologique, qui échappe à la prose, encombrée, elle, par les détails et les particularités de l'histoire, intéressée par des personnages qui ne sont que les instruments de Dieu ${ }^{48}$.

Ce dernier exemple nous amène à considérer à présent les inscriptions à caractère prophétique. Ce sont les plus nombreuses et les plus frappantes pour le lecteur mais 
surtout pour les personnages du roman qui le plus souvent ne les comprennent pas, alors que le lecteur peut parfois en deviner le sens. Une série est constituée par les multiples textes qui surgissent sur le perron du Franc-Palais. Le premier texte apparait lors de la fête célébrant le mariage de Cerse et Béthidès et annonce la prochaine invasion des Romains, la destruction de la Grande-Bretagne et enfin sa restauration (PF, IV-1, p. 399). Aucun personnage, et le lecteur non plus du reste, ne peut savoir que l'aigle noir qui doit se reposer sur le perron est Jules César. Ce n'est qu'après la bataille du Franc-Palais que le narrateur nous donne la clé de l'énigme : Jules César, s'étant assis sur le perron, lit l'inscription et comprend qu'elle s'applique à lui puisqu'il porte un écu d'or à aigle noir. En revanche, il ne peut encore comprendre la deuxième partie, celle qui prophétise la restauration du royaume (PF IV-1, p. 644-45). Or ce même perron réapparait plus avant dans l'histoire, mais porteur d'un message différent, bien que le roman prenne la peine de rappeler la teneur de la précédente inscription. La terrible prophétie concernant la ruine du pays s'étant réalisée, la première inscription a été remplacée par une autre qui annonce à long terme la grandeur retrouvée du royaume de Grande-Bretagne et à court terme l'arrivée de deux chevaliers qui adouberont deux écuyers (PF IV-2, 796). Un peu plus tard, une troisième inscription peut se lire qui promet, outre la restauration à venir du royaume, la venue d'un chevalier «Ours» qui adoubera les jeunes gens du royaume (PF IV-2, p.924-25). Enfin une dernière inscription vient remplacer celle-ci et annoncer à Gallafur que prochainement une épée merveilleuse sera fichée dans le perron et que seul le "chef du lignaige / Perceforest » pourra l'en arracher avant de devenir un puissant roi qui restaurera le royaume.

Par ce jeu d'écritures successives sur le perron, un pan de l'histoire à venir de la Grande-Bretagne se dévoile. Ce qui se révèle surtout à cette occasion, c'est que dans cette histoire chaotique, désespérante pour ceux qui en sont les acteurs, une force est à l'œuvre, le Dieu souverain que Perceforest et ses hommes ont commencé à vénérer sans le connaître. Car c'est Dieu qui écrit les messages sur le perron; c'est lui qui envoie à ses amis ces signes. L'ermite Dardanon, qui a décidé dès le début du roman d'abandonner les dieux antiques et de se tourner vers ce dieu inconnu, interprète pour Perceforest les prodiges qui se sont produits lors du mariage de son fils : l'irruption d'un aigle noir qui fait tomber la tête vermineuse, la prophétie de cette dernière, les serpents qui s'échappent de la tête et mettent en pièce les chapeaux des convives. Pour Dardanon, ce sont des avertissements envoyés par Dieu dont l'esprit Zéphir est le messager : « de ceste mervelle je ne sçay jugier se elle advint par le fait d'aucun mauvais angele ou par le vouloir du Dieu Souverain, comment que je seroie plus tost d'oppinion que ce soit signe du Dieu Souverain selon ce que j'ay veu es signes celestiaulx » (PF IV-1, p. 544-45). Les prophéties concernant le royaume de Perceforest sont en effet incluses dans un ensemble de signes extraordinaires annonçant un événement bien plus considérable : la venue du Christ. L'histoire de la Grande-Bretagne se comprend donc à la lumière de cette autre histoire qu'est l'histoire chrétienne. Dieu veut la restauration et l'épanouissement du royaume de Grande-Bretagne, la naissance de peuples qui suivront sa loi, comme les Irlandais de la reine Flamine, fille de Flora et d'Aroés, chez qui Ourseau trouve un temple à la gloire du Dieu souverain. Une inscription en vers explique que le temple est dédié à ce nouveau Dieu tout-puissant et engage le visiteur à l'adorer (PF IV-2, p. 815). Cette conversion au Dieu Souverain avant même l'Incarnation est un signe des Temps. 
41

r, pour se manifester et pour révéler une partie de l'avenir, Dieu choisit l'écriture en vers. Le sacré ne s'exprime pas en prose. Les humains le savent bien, qui choisissent parfois de s'adresser aux dieux sous une forme poétique. Brutus le premier prie la déesse Diane en vers (PF I, p. 76). Sans doute l'auteur du roman se contente-t-il ici de traduire Geoffroy de Monmouth qui rédige également cette prière en vers. Mais il aurait pu la traduire en prose ${ }^{49}$. La longue oraison de l'ermite Dardanon, surprise par Perceforest, est également en vers (PF I, p. 418-20). Le Lai des Jeunes Filles au Dieu des Désiriers (PF IV-2, p. 1100-105) n'est autre chose qu'une prière chantée. Les dieux répondent souvent en vers: ainsi Diane, chez Geoffroy comme dans le Perceforest, ou parfois le Dieu des Désiriers (PF IV-2, p. 914). Vénus prodigue ses conseils à Troylus sous la forme de deux huitains dont le sens érotique sera explicité par l'aventure du héros (PF III-3, p. 80).

42

Un épisode met particulièrement en évidence la valeur sacrée du vers. Ourseau et Passelion découvrent deux chevaliers enferrés, punition qui remonte à la toute première époque, celle où les demoiselles de la forêt immortalisaient les exploits des nouveaux chevaliers du royaume contre Darnant en érigeant des statues de chair. L'insolite spectacle de ces chevaliers enferrés est accompagné d'une inscription en prose : «O tu, chevalier, qui ces deux chevaliers regardes, ne les tiens pour mors, car se tu as le hardement de veillier jusques a mynuit icy, tu les verras en point de jouter; et se a eulx joustes, de noble aventure te pourras bien vanter. ( (PF IV-2 1109-10). Il n'en faut pas plus pour convaincre Passelion de tenter l'aventure malgré les avertissements répétés de Zéphir qui le lui déconseille. En effet, l'inscription n'est qu'un piège tendu par les suppôts de Darnant pour mettre en danger les bons chevaliers. Or à cette inscription en prose fait pendant une inscription en vers qui rétablit la vérité en expliquant l'origine de la punition subie par les deux corps. Le poème s'achève sur une question purement rhétorique destinée à remplir d'effroi le spectateur: «Regarde, chevalier amant,/Est la vengeance souffissant?»(PF IV-2, p. 1110). Mais la valeur et la fonction de la question sont faussées par l'inscription en prose ajoutée plus tard et par la déclaration d'une mystérieuse voix qui engage encore les chevaliers de passage à veiller auprès des cadavres : «Seigneurs chevaliers, se l'amende vous samble petite et cuer avés de l'amender, soyés cy a l'eure de minuit. Vous les avrés presents, sy les tuez la seconde fois.» Incapables d'interpréter correctement les messages contradictoires qu'ils ont déchiffrés ou entendus, Passelion et Ourseau tentent l'aventure pour leur plus grande honte. Darnant et son lignage ont compris le pouvoir de l'écriture et pour égarer les bons chevaliers, émaillent le paysage d'inscriptions semblables à celles des dames de la forêt. À ce stade du roman, ils n'ont cependant pas encore maîtrisé l'écriture en vers. Si l'impétueux et un peu sot Passelion avait examiné plus attentivement les deux conseils, il aurait deviné lequel émane de Dieu, lequel émane des puissances mauvaises. Plus tard, au livre VI, vient un autre chevalier aussi vaillant mais plus réfléchi, Gallafur, élu pour porter le coup fatal à Darnant. Traqués, les mauvais esprits tentent une ultime manœuvre pour résister à leur vainqueur : ils se mettent à écrire en vers des messages tantôt provocateurs, pour les chevaliers de la trempe de Passelion, ${ }^{50}$ tantôt dissuasifs, pour Gallafur particulièrement. Au lieu de promettre une noble aventure, ils annoncent la honte de celui qui osera s'approcher de la tombe de Darnant :

"O chevallier, garde ou tu vas

$\mathrm{Ne}$ passes point oultre ce pas 
Pour ceste aventure aciever

Se ne veulx ton corps desciever

Ou mettre a mort par aultre voie.

Force n'y vault c'un fil de soie

Vauldroit a ung grant vent abatre.

Fol est que se y vient debatre. (PF VI, Ars. ms 3493, fol. 16ํo).

Certaines inscriptions cherchent à écarter Gallafur des aventures qu'il doit mener à terme en tentant de le convaincre qu'un autre chevalier les a déjà achevées. ${ }^{51}$ Elles figurent à côté d'écrits en vers émanant, eux, de Dieu, de sorte que le passant et le lecteur ne savent plus avec certitude à qui attribuer les unes et les autres. Imitant la rhétorique divine, les mauvais esprits sèment la confusion et menacent tout le système d'autorité mis en place par le Perceforest. La gravité de ce détournement sacrilège est à la mesure de la panique qui s'est emparée d'eux devant l'inexorable avancée de leur ennemi. Gallafur cependant n'est pas le lecteur ordinaire. Inspiré par Dieu et protégé par ses armes marquées du signe de la croix, il sait distinguer les bons conseils des faux. Il donne du reste un petit cours d'interprétation à son compagnon Blanor : « vous povez maintenant veoir comment les mauvais esperitz ont deceu par cy devant les vaillans preudommes en armes et en chevallerie par leurs vers. " (PF VI, Ars. ms 3493, fol. 30roํ). Non seulement il détruit définitivement Darnant et son lignage mais encore il sauve le système du roman en annulant les inscriptions trompeuses et en rétablissant la confiance dans le vers.

Prose et vers ne sont pas en concurrence dans le Perceforest. Tous deux visent à garantir la vérité du texte. Si l'histoire s'écrit naturellement en prose, elle a besoin du discours poétique pour la sortir d'un quotidien sans perspective et l'orienter. Langage du sacré, l'écriture en vers manifeste en effet la présence et l'action de Dieu dans l'histoire des hommes, présence sans laquelle les aventures de l'humanité s'enchaîneraient dans une pure succession sans signification. Dieu et le discours poétique empêchent l'histoire de sombrer dans la simple chronique. Cette alliance salutaire du vers et de la prose, c'est le rôle du ménestrel de la proclamer et de la maintenir. Clerc, héraut et ménestrel, Ponchonnet incarne la réconciliation de deux modes d'écriture que l'on avait indûment spécialisés, l'un, la prose, dans le discours historique à prétention réaliste, l'autre, le vers, dans le discours de l'émotion.

\section{NOTES}

1.Perceforest, première partie, éd. Jane H. M. Taylor, Genève : Droz, 1979; quatrième partie, éd. Gilles Roussineau, Genève : Droz, 1987, 2 volumes ; troisième partie, éd. Gilles Roussineau, Genève, Droz, 1988-1993, 3 volumes. PF = Perceforest, le chiffre romain indique la partie, le chiffre arabe le volume, le dernier nombre la page. Pour la partie non éditée du roman, j'utilise le manuscrit en 12 volumes de l'Arsenal : Paris, Arsenal, 3483-3494. Chaque partie est contenue dans deux volumes. Partie I : mss 3483 et 3484 ; partie II : mss 3485 et 3486 ; partie V : mss 3491 et 3492 ; partie VI : mss 3493 et 3494. 
2.Sur l'émergence de l'historiographie en prose française voir Gabrielle M. Spiegel, Romancing the Past. The Rise of Vernacular Historiography in Thirteenth-Century France, Berkeley, University of California Press, 1993, en particulier l'introduction.

3.L'expression « insertions lyriques » ne convient qu'imparfaitement. Si elle peut à la rigueur s'appliquer aux lais et chansons (avec des réserves similaires à celles qu'émet E. Baumgartner à propos des pièces lyriques du Tristan en Prose. Voir La Harpe et l'Epée, Tradition et Renouvellement dans le Tristan en Prose, Paris, SEDES, 1990, p. 118), elle devient totalement inappropriée pour les épitaphes et inscriptions en vers qui n'ont le plus souvent rien de lyrique et ne constituent pas des ajouts décoratifs.

4.Dans un article très intéressant (« Récit historique et discours poétique dans L'Estoire de la guerre des Ibelins contre les Impériaux de Philippe de Novarre », Le Moyen Age, 1997, $\mathrm{n}^{\circ} 1$, pp. 67-81), C. Aslanoff pose des questions similaires. Les réponses qu'il offre sont différentes des miennes. Mais le texte qu'il analyse est un authentique texte historique. Le problème de la véridicité ne s'y pose donc pas de la même manière et le statut du vers ne peut être le même. Dans le Perceforest, prose et vers sont tous deux au service du voir dire.

5.J. H. Taylor dans l'introduction à son édition du livre I amorce l'étude du prologue (p. 33 et 34 en particulier). Ses abondantes notes permettent également de mesurer le travail de traduction et de remaniement auquel l'auteur du Perceforest s'est livré sur les œuvres de Geoffroy de Monmouth et Orose. Voir aussi son article : «The Fourteenth Century : Context, Text and Intertext ", dans The Legacy of Chrétien de Troyes, éd. N. Lacy, K. Busby et D. Kelly, Amsterdam, Rodopi, 1987, vol. 1, en particulier pp. 275-282. 6.Orose, Historiae Adversum Paganos, Libri VII, éd. C. Zangemeister, Vienne, 1882 ; Geoffroy de Monmouth, Historia Regum Britanniae, éd. E. Faral, La Légende Arthurienne, Etudes et Documents, Première partie, t. III, Paris, Champion, 1969. Voir aussi l'introduction et les notes de J.H.M. Taylor, au livre I de Perceforest.

7.Voir B. Lacroix, Orose et ses idées, Montréal-Paris, Institut d'Etudes Médiévales-Vrin, 1965. Voir en particulier sur la géographie, «fondement essentiel à l'histoire écrite », p. 56 (dans «Orose et sa conception de l'histoire»). Sur Geoffroy de Monmouth, voir E. Faral, La Légende Arthurienne, Paris, Champion, 1929, t. 1, p. 68 (Geoffroy emprunte sa description de la Grande-Bretagne à ses propres sources : Gildas et Bède).

8.Orose, Historiae Adversum Paganos, 28-77 : Haec insula habet in longo milia passuum DCCC, in lato milia CC. Geoffroy de Monmouth, p. 27.

9.Dans les manuscrits BN fr 106 (fol. 2roo) et BN fr 345 (fol. 2ro) le nom de Darès, ainsi parfois que celui d'Orose, apparaît en retrait du texte, (en rouge dans fr 106 où le nom de Darès est immédiatement suivi de la rubrique du chapitre), comme une addition. Dans le ms Ars. 3483 fol. 12ro , la description de l'Irlande est introduite par : « Oroses dist que... ». Le nom de Darès n'apparaît pas. Dans son édition, J. H. M. Taylor reproduit également les noms en fin de ligne.

10. Roman de Brut, éd. Ivor Arnold, Société des Anciens Textes Français, Paris, 2 volumes, 1938, vv. 1064-70. Voir aussi Roman de Rou, éd, A.J. Holden, Paris, SATF, 3 vol. 1970-73, vv. 6415-18.

11. Sur ce point, voir J. Monfrin, «Traductions au Moyen Age », dans L'Humanisme médiéval dans les littératures romanes du XIIe au XIVe siècle, éd. A. Fourrier, Paris, Klincksieck, 1964.

12.Au point que, comme le note J.H. M. Taylor, des chapitres du roman ont trouvé leur place dans des chroniques du XIV ${ }^{e}$ siècle ou plus tardives (intro. de PF I, p. 25-27, et 
note 22 p. 36). Voir aussi la réaction du marquis de Paulmy citée par J. Lods dans son étude : Le Roman de Perceforest, Genève-Lille, Droz-Giard, 1951, p. 37.

13. Historia Regum Britanniae p. 51.

14. En particulier PF, I pp. 90-93, 99.

15. Voir aussi L.F. Flûtre, «Etudes sur le Roman de Perceforêt (huitième et dernier article) ", Romania, 91, 1970, p. 214. Ms. Ars. 3494 fol. 329ro à 331.

16. Sur le motif de l'armoire, voir E. Baumgartner, «Armoires et Grimoires », dans De l'Histoire de Troie au livre du Graal; le temps, le récit (XII ${ }^{-} \mathrm{XIII}^{e}$ siècles), Orléans, Paradigme, 1994.

17.Sur cette partie du prologue voir S. Huot, «Chronicle, Lai and Romance: Orality and Writing in the Roman de Perceforest ", dans Vox intexta: Orality and Textuality in the Middle Ages, éd. A. Nick Doane et Carol Braun Pasternak, Madison, University of Wisconsin Press, 1991, pp. 203-223.

18.Date fausse, le mariage ayant eu lieu en 1308 . Voir J. Taylor, « The Fourteenth Century », art. cit. p. 280.

19.La Compilation de Rusticien de Pise (ms BN fr. 340) est également placé sous le patronage, plus direct il est vrai, d'un Édouard d'Angleterre, Édouard I ${ }^{\text {er }}$, qui aurait possédé un livre contenant le roman de chevalerie. Voir C. E. Pickford, L'Évolution du Roman Arthurien en prose vers la fin du Moyen Age, Paris, Nizet, 1959, p. 206. L'auteur de Perceforest a-t-il été influencé par le prologue de la Compilation?

20.J. H. M. Taylor se demande même dans l'introduction à son édition si la supercherie imaginée par l'auteur du Perceforest aurait pu l'être avec la complicité de la Cour de Hainaut (PF I, p. 33).

21.C'est celle de Perceforest, qu'il a déposé lui-même dans « l'aumaire » avec le livre des chroniques et une lettre explicative, comme nous le révèle la sixième partie du roman (voir infra p.11). Avant la découverte de l'abbé en effet, le livre, caché par Perceforest lors de l'invasion des Romains, est découvert une première fois par les descendants de Perceforest. Le chevalier Gallafur voit d'abord en rêve l'armoire et ses trésors (PF IV-2, 1019) ; puis la jeune Alexandre-Fin de Liesse ouvre la porte de l'armoire et exhume les précieux documents (PF VI, chap. XXVI, ms Ars. 3493 fol. 178-179). Sur une interprétation de cette couronne, voir S. Huot, «Chronicle, Lai and Romance... », art. cit. pp. 220-21.

22.Je remercie E. Baumgartner de m'avoir suggéré ce rapprochement qui m'avait échappé.

23.C'est le roi Gallafur qui redoutant l'invasion danoise a pris soin d'enfermer pour la seconde fois (voir infra) dans l'armoire le livre des chroniques. Voir J. Taylor, « The Fourteenth Century ", art. cit. p. 280.

24.G. Roussineau les édite partiellement toutes les cinq dans l'introduction au livre III, tome I, p. VII-IX, et s'interroge sur leur auteur : sont-elles l'œuvre du romancier ou l'ajout d'un remanieur? Bien que l'éditeur ne se prononce pas formellement, il souligne que « la distribution de la manière romanesque en six volumes d'égale épaisseur n'a rien d'une division arbitraire »(p. IX). Les préfaces sont donc parties intégrales du roman et leur analyse est indispensable pour tenter de déterminer le statut du narrateur.

25.Voir J. Taylor, «The Fourteenth Century », art. cit. p. 282.

26.Le nom de Crésus apparaît dans les préfaces du livre III (PF III-1, p. 1), IV (PF IV-1,

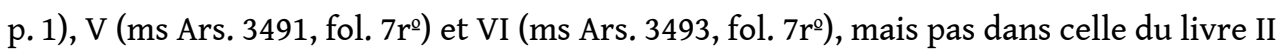


où le narrateur-éditeur s'attribue plus nettement le travail de conteur : «quand j'ay eu achevé le tournoy du gentil roy Gafiffer et aussi comment le conquerant Alixandre s'estoit party d Scidrac [...\} je m'advisay de faire fin au premier volume. » (ms Ars. 3485, fol. $\left.5 r^{\circ}\right)$.

27.Par exemple, Cresus « fut commis de mettre en memoire et par ordre toutes les aventures qui avindrent en la Grant Bretagne depuis que Perceforest fut couronné roy jusques à sa fin » (PF III-1, p. 221); « Le noble clercq Cresus ne voult point oublier de mettre en ces cronicques l'adventure de la belle Neronés et celle du Chevallier Doré quant il en sceut la verité » (PF III-2, p. 200-201). Voir aussi PF III-1, p. 327.

28.Voir aussi L. F. Flûtre, «Etudes sur le Roman de Perceforest (huitième et dernier article) », Romania p. 207.

29.Voir la fin de La Queste del Saint Graal par exemple : « li rois fist avant venir les clers qui metoient en escrit les aventures aus chevaliers de laienz. Et quant Boorz ot contees les aventures del Seint Graal telles come il les avot veues, si furent mises en escrit et gardees en l'almiere de Salebieres. » éd. A. Pauphilet, Paris, Champion, 1978, p. 279-80. Même technique dans le Lancelot en prose (voir éd. A. Micha, t. VIII, Genève, Droz, p. 488-89).

30.Sur ce point, voir E. Baumgartner, « Une structure arborescente, les proses du Graal », dans Les Modèles de la création littéraire, Littérales nº 5, Publidix, Nanterre, 1989. 31.Sur le vers chargé de dire par exemple la "vérité de l'être », voir E. Baumgartner, $L a$ Harpe et l'Épée, op. cit. pp. 114-124.

32.J. Taylor a montré que le narrateur de Perceforest intervient aussi personnellement, et en prose, pour « historier la chronique », c'est-à-dire pour orienter la lecture et souligner les liens de cause à effet qui pourraient avoir échapper au lecteur («The Fourteenth Century ", art. cit. pp. 283-85).

33.Les Pièces lyriques du Roman de Perceforest, Genève, Droz, 1953 et Le Roman de Perceforest, op. cit.

34.Chapitre 42. Voir L. F. Flûtre, «Études sur le Roman de Perceforest (huitième et dernier article) », p. 194. Ms. Ars. 3492 fol. $457 v^{\circ}$. Le texte du lai suit du fol. 458 au fol. $467 \mathrm{v}^{\circ}$.

35.Roman de Tristan en Prose, t. 1, éd. Ph. Ménard, Genève, Droz, 1987, pp. 226-247. Sur l'ensemble de cet épisode, voir E. Baumgartner, La Harpe et l'Épée, op. cit. pp. 121-124.

36.Voir aussi PF III-1, pp. 69-71 où Pergamon relate en détail la " cérémonie des vœux " et ce qui s'en est suivi ; et III-1, p. 257.

37.PF V, chap. 30. Ms. Ars. 3493 fol. $197 \mathrm{v}^{\circ}$ à $198 \mathrm{r}^{\circ}$.

38. Voir aussi le lai du Dieu des Desiriers aux Pucelles. Ourseau, qui ne connaît bien sûr rien du Chevalier au Dauphin, entend des jeunes filles chanter les mérites de ce dernier dans un lai (PF, IV-2, p. 913, texte du lai : PF IV-2 1100-1105).

39.Voir aussi L.F.Flûtre, «Etudes sur le Roman de Perceforest (huitième et dernier article) », p. 205.

40.Le roi des ménestrels qui vient rendre visite à Lyonnel malade au livre III (donc dans la partie qui raconte les douze tournois) est Ponchonnet (PF III-2, p. 13).

41.Sur le rôle du ménestrel dans la conservation du passé, voir aussi S. Huot, "Chronicle, Lai, Romance... », art. cit.

42.Dans le prologue du livre V, Crésus est appelé « le sage clerc et le venerable poete » (ms Ars. 3491, fol. 7ro $)$. 
43.Ms. Ars. 3493 fol. $198 \mathrm{v}^{\circ}$. Avant d'être chargé de la rédaction des chroniques du règne de Gallafur, Ponchon est choisi pour lire le livre de Perceforest à toute la cour.

44. Voir G. Roussineau, note au paragraphe I de la quatrième partie (PF IV-2, p. 1139).

45.Voici par ex. la liste des inscriptions versifiées dans PF IV-2 :

p. 815, Ourseau au temple de la reine Flora: 12 vers octosyllabiques sur deux rimes; p. 914 , Ourseau au temple du Dieu des Desiriers : 5 alexandrins sur une rime ; p. 924-925, inscription sur le perron du Franc-Palais: 8 couplets d'octosyllabes à rimes plates; p.947-48, le demoisel merveilleux au pont de l'épée : deux insertions en vers octosyllabiques à rimes plates, l'une de 10 vers, l'autre de $6 ;$ p. 1019, inscription sur le perron du Franc-Palais : 10 décasyllabes à rimes plates; p.1110, inscription concernant les deux chevaliers enferrés : 10 octosyllabes à rimes plates.

46.Voir mon article «Aroés l'illusionniste », Romania, p. 11

47.Le vieil homme explique en effet au Chevalier Doré qu'il était dans sa jeunesse un riche et vaillant chevalier mais que devenu veuf et sans enfants, il avait décidé de se retirer dans un lieu solitaire pour s'occuper du salut de son âme. Il a fait bâtir près de son ermitage un temple dédié à Vénus.

48.Pour une analyse détaillé de l'épisode, voir " Aroés l'illusionniste », art. cit.

49.Wace dans son Brut choisit quant à lui d'éliminer purement et simplement le texte de la prière.

50.Voir en particulier fol. 30ro (Ars. 3493) qui commence ainsi : «Bien pert que la chevallerie/De Bretaigne est bien empirie/Quant cest chemin adventureuz/Est si laissié en paix pourreuz./Ce sont moisnes, ce croy, de cloistre/Qui laissent icy tant l'erbe croistre/ » etc.

51.Voir par ex. l'inscription au fol. 32 (ms. Ars. 3293). Gallafur, convaincu d'être arrivé trop tard, va céder au désespoir quand son ami Blanor, qui a bien profité de ses leçons, le réconforte: « par adventure est cecy aucune deception que les mauvais esperitz ont fait pour vous destourner la voie.»

\section{AUTEUR}

\section{MICHELLE SZKILNIK}

Université de Nantes 